\title{
High-responsivity graphene infrared photodetectors based on photogating
}

\section{Shinpei Ogawa}

Shinpei Ogawa, "High-responsivity graphene infrared photodetectors based on photogating," Proc. SPIE 11525, SPIE Future Sensing Technologies, 1152503 (8 November 2020); doi: 10.1117/12.2583742

SPIE. Event: SPIE Future Sensing Technologies, 2020, Online Only 


\title{
High-responsivity graphene infrared photodetectors based on photogating
}

\author{
Shinpei Ogawa \\ Advanced Technology R\&D Center, Mitsubishi Electric Corporation, 8-1-1 Tsukaguchi-Honmachi, \\ Amagasaki, Hyogo 661-8661, Japan
}

\begin{abstract}
Graphene is a promising material for next-generation high-performance photodetectors because of its fast response, broadband photodetection (from the ultraviolet region to the terahertz region), mechanical and chemical stability, flexibility, and low manufacturing cost. We developed high-responsivity graphene infrared (IR) photodetectors based on field-effect transistors (FETs) with photogating that operate in the middle- or long-wavelength IR (MWIR or LWIR) region. The photogating effect is induced by a photosensitizer located in the vicinity of graphene. The photosensitizer generates a voltage change via incident light, which modulates the carrier density of graphene and produces an extremely large differential output current. This effect, unique to graphene, is attributed to its high carrier mobility and single-atom thickness. As photosensitizers, InSb and $\mathrm{LiNO}_{3}$ were used for the MWIR and LWIR regions, respectively. However, graphene FET-based structures inevitably produce a large dark current because graphene has no bandgap. This degenerates photodetector performance and prevents the construction of IR image sensors using conventional readout integrated circuits. To overcome this problem, a graphene/InSb heterostructure is proposed. It exhibits both a low dark current and a high responsivity by amplifying injected photocarriers via photogating in the MWIR region. These results can be applied to other wavelength regions and could contribute to the development of high-performance graphene-based IR image sensors and next-generation optoelectronic devices.
\end{abstract}

Keywords: graphene, 2D materials, infrared, photodetectors, photogating

\section{INTRODUCTION}

Graphene, an atomically thin material with a carbon-based honeycomb lattice, has a unique electronic band structure with Dirac cones ${ }^{1,2}$. Because of this band structure, graphene has excellent properties, including high carrier mobility, a broadband photoresponse, and mechanical and chemical stability ${ }^{3,4}$. Graphene is flexible ${ }^{5}$ and can be fabricated at low cost using methods such as chemical vapor deposition ${ }^{6}$. These advantages make graphene suitable for next-generation optoelectronic devices such as photodetectors (PDs). The development of high-performance ultrabroadband PDs with a fast response time is expected ${ }^{7,8}$. However, graphene has a low absorbance $(\sim 2.3 \%)$. Various methods have been proposed to enhance its responsivity, including those based on hetero-electrodes ${ }^{9,10}$, optical cavities ${ }^{11,12}$, plasmonic structures $^{13-17}$, nano-patterned graphene for graphene plasmons ${ }^{18-22}$, the bolometric effect ${ }^{23}$, PN junctions ${ }^{24}$, quantum $\operatorname{dots}^{25-27}$, heterostructures with semidonductors ${ }^{28-32}$, and photogating ${ }^{33-37}$. Among them, photogating is the most effective method for achieving ultra-high responsivity, which cannot be achieved using conventional technologies.

There has been growing demand for high-performance infrared (IR) PDs for applications in security, defense, the automotive industry, and medicine. To meet this demand, we developed high-responsivity graphene IR PDs based on photogating. In this paper, we report the detailed mechanism of the photogating and the developed graphene PDs with various structures and photosensitizers.

\section{PHOTOGATING}

Figure 1 shows the photogating mechanism. Figure 1(a) shows a schematic diagram of graphene field-effect transistor (FET)-based PDs. If a photosensitizer that can generate a voltage change $\left(\mathrm{V}_{\mathrm{ph}}\right)$ when irradiated by IR light is used as a substrate, the back-gate voltage is modulated by $\mathrm{V}_{\mathrm{ph}}$, changing the Fermi level of the graphene, as shown in Fig. 1(b). Consequently, as shown in Fig. $1(\mathrm{c})$, the $\mathrm{V}_{\mathrm{bg}}-\mathrm{I}_{\mathrm{d}}$ curve is shifted. The differential output current $\left(\triangle \mathrm{I}_{\mathrm{ph}}\right)$ is extremely large

SPIE Future Sensing Technologies, edited by Masafumi Kimata, Joseph A. Shaw, Christopher R. Valenta, Proc. of SPIE Vol. 11525, 1152503 - @ 2020 SPIE - CCC code: 0277-786X/20/\$21 · doi: 10.1117/12.2583742 
because it is proportional to the gradient of the $\mathrm{V}_{\mathrm{bg}}-\mathrm{I}_{\mathrm{d}}$ curve, which corresponds to the high carrier mobility of graphene. Because graphene is atomically thin, a small voltage change can produce a large modulation of carriers. The $\Delta \mathrm{I}_{\text {ph }}$ of graphene-based PDs is at least 10 times larger than that of conventional quantum-type PDs. The photosensitizer determines the operating wavelength and response time of graphene-based PDs.
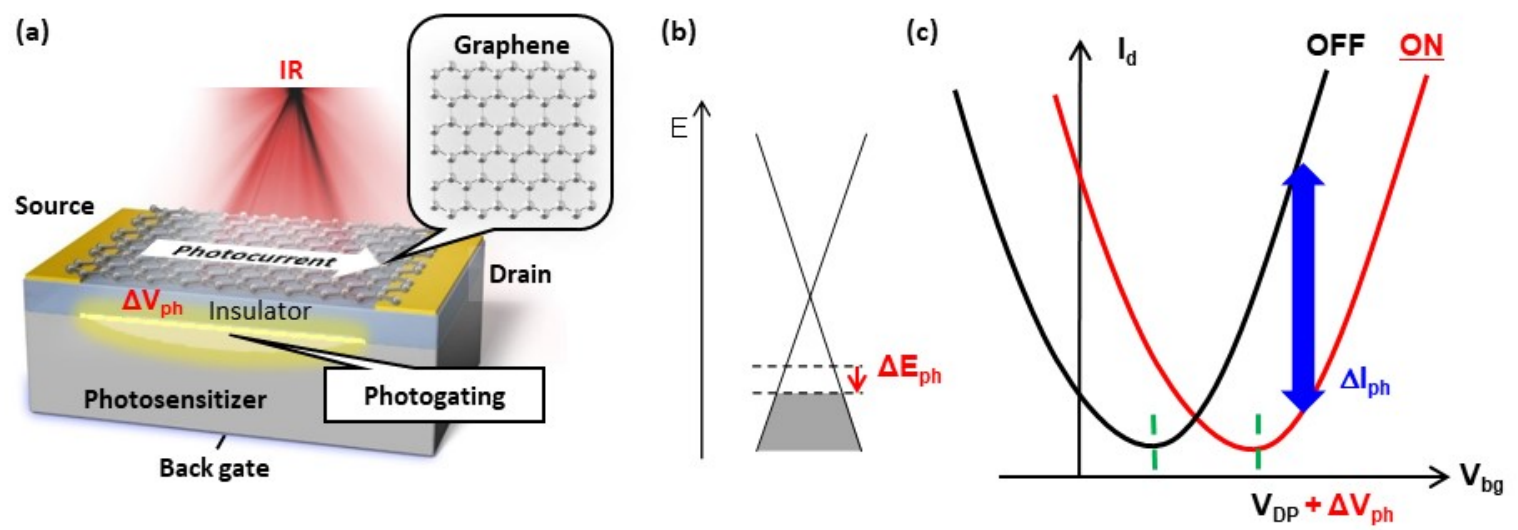

Figure 1. (a) Schematic diagram of photogating mechanism for graphene FET-based PDs. (b) Fermi level shift of graphene caused by back-gate voltage change. (c) $\mathrm{V}_{\mathrm{bg}}-\mathrm{I}_{\mathrm{d}}$ curve shift caused by photogating.

\section{GRAPHENE FET-BASED PHOTODETECTORS}

Figure 2 shows the developed graphene FET-based PDs based on photogating. $\mathrm{Si}^{33}{ }^{38}, \mathrm{InSb}^{34,37}$, and $\mathrm{LiNbO}_{3}{ }^{35}$ were used for the visible (VIS) and near-IR (NIR), middle-wavelength IR (MWIR), and long-wavelength IR (LWIR) regions, respectively. Si has a bandgap that spans the VIS region and part of the NIR region, and the InSb bandgap spans the MWIR region. $\mathrm{LiNbO}_{3}$ exhibits a pyroelectric effect in the LWIR region. These materials can therefore produce a photogating effect in these wavelength ranges.

All structures consisted of an insulator layer formed on the substrate as a photosensitizer, onto which a graphene FET was fabricated with source-drain electrodes.
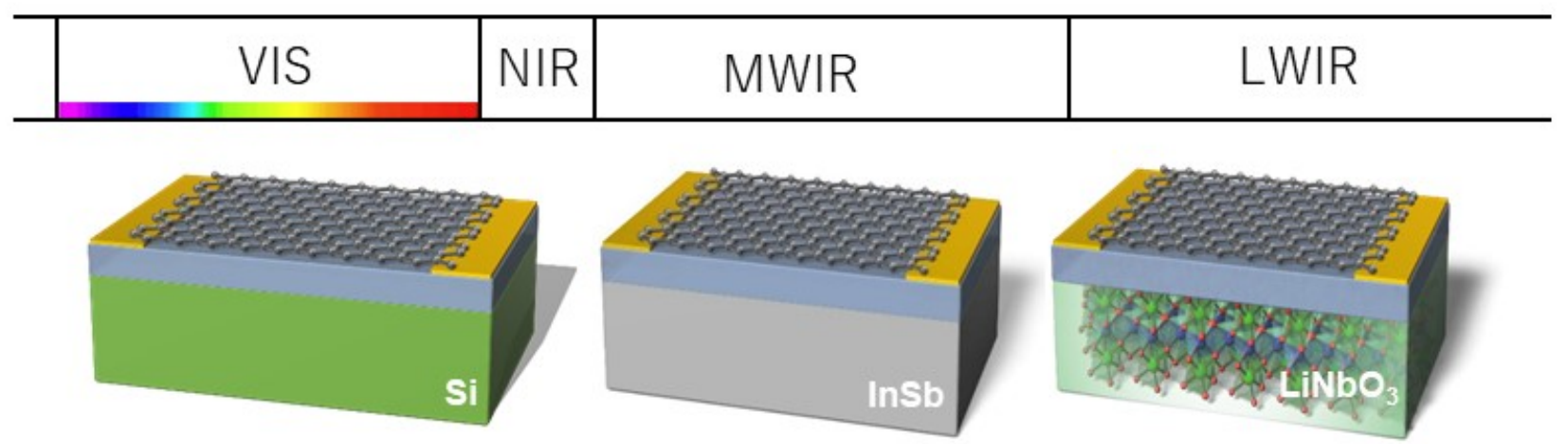

Figure 2. Graphene FET-based photogating PDs using Si (VIS and NIR), InSb (MWIR), and $\mathrm{LiNbO}_{3}$ (LWIR) as photosensitizers. 
Figure 3 shows a comparison of the photoresponse of graphene-based IR PDs using InSb and Si substrates, respectively, in the MWIR region at $77 \mathrm{~K}$. Only InSb produces a voltage change and thus a photogating effect, because the Si bandgap does not span the MWIR region. The photoresponse with photogating was approximately 100 times larger than that without photogating.

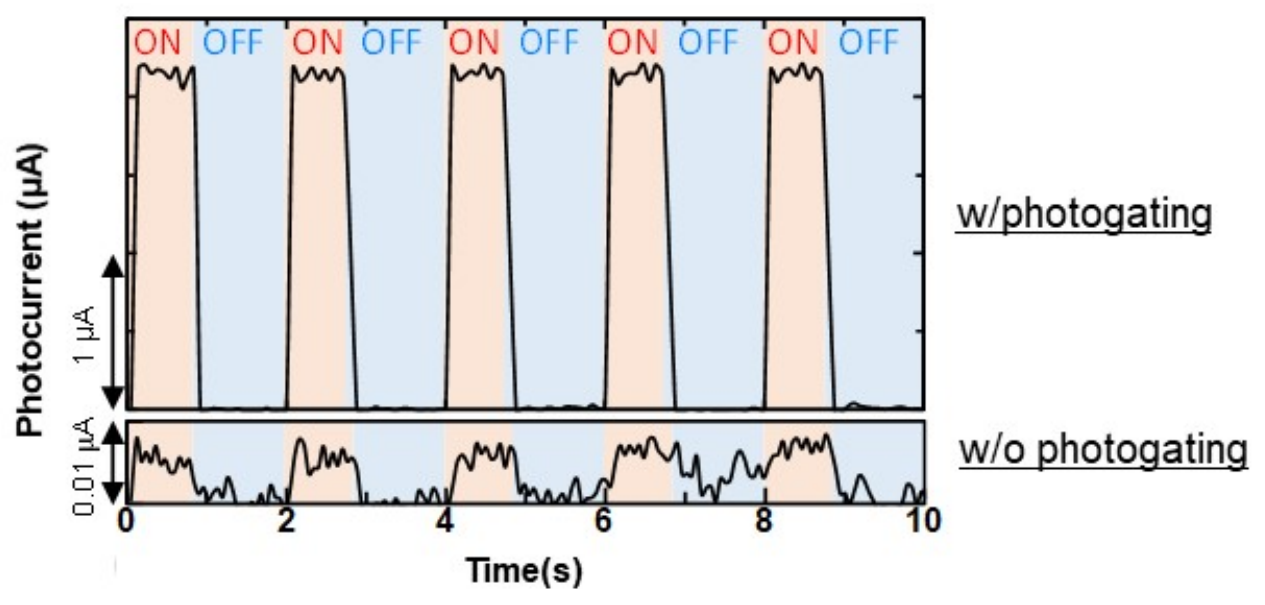

Figure 3. MWIR photoresponse of graphene IR PDs with InSb (top) and Si (bottom) substrates.

Ultra-high responsivities of $2.86 \times 10^{5}, 321$, and $446 \mathrm{~A} / \mathrm{W}$ were obtained with $\mathrm{Si}^{38}, \mathrm{InSb}^{37}$, and $\mathrm{LiNbO}_{3}{ }^{35}$ as the photosensitizer in the VIS, MWIR, and LWIR regions, respectively. The corresponding quantum efficiencies of all PDs exceeded $100 \%$. These results are direct evidence of the photogating effect. In addition, room-temperature operation can be realized in the $\mathrm{LWIR}$ region owing to the pyroelectric effect of $\mathrm{LiNbO}_{3}$.

\section{PHOTODETECTORS BASED ON GRAPHENE/SEMICONDUCTOR HETEROSTRUCTURE}

The previous section showed that graphene FET-based IR PDs with photogating exhibit ultra-high responsivity. However, graphene-based FET structures inevitably produce a large dark current because graphene has no bandgap. To address this issue, we developed a graphene/semiconductor heterojunction with photogating, where graphene and the semiconductor are in direct contact. In this contact region, a Schottky barrier forms owing to the difference in Fermi level between graphene and the semiconductor ${ }^{39}$. The dark current is expected to be reduced by this barrier, which operates like a diode. PDs with this structure are hereafter denoted as diode-type PDs.

Figures 4(a) and 4(b) show the mechanism of photogating in graphene FET-based and diode-type PDs, respectively. As shown in Fig. 4(b), a graphene-based diode-type PD comprises a contact region and a graphene channel. In the contact region, where graphene and the semiconductor are in direct contact, photocarriers are injected from the substrate to graphene. The graphene channel forms on the insulator layer on the substrate, where photogating can occur. Therefore, diode-type PDs exhibit both high responsivity and a low dark current. 
(a)

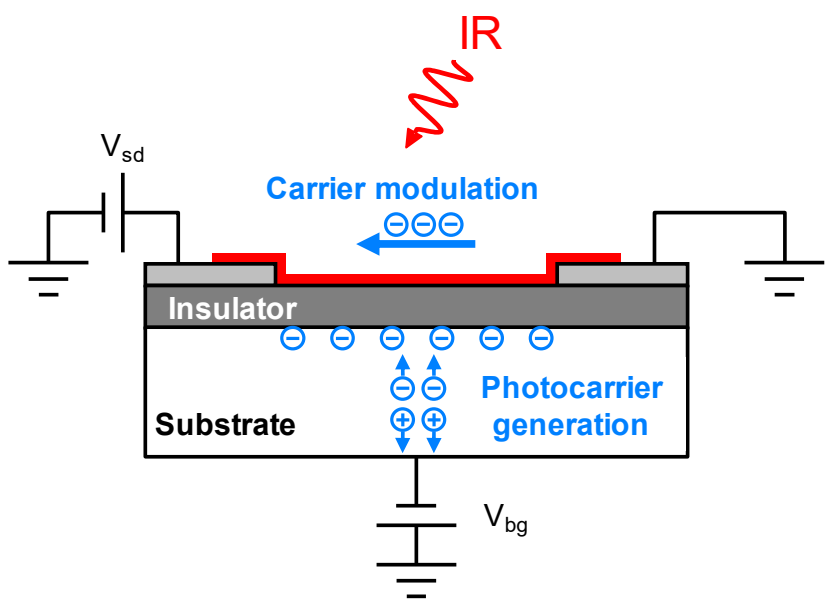

(b)

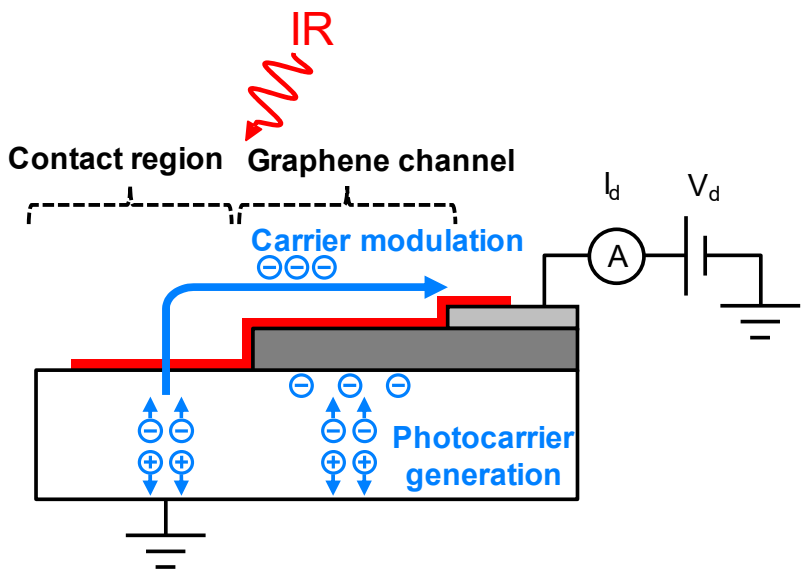

Figure 4. Comparison of operation mechanism of graphene (a) FET-based and (b) diode-type PDs with photogating.

Figures 5(a) and 5(b) show a schematic diagram of the developed graphene/InSb heterojunction MWIR $\mathrm{PD}^{30}$ and a comparison of the photoresponse between graphene FET-based and diode-type MWIR PDs with an InSb substrate, respectively.

(a)

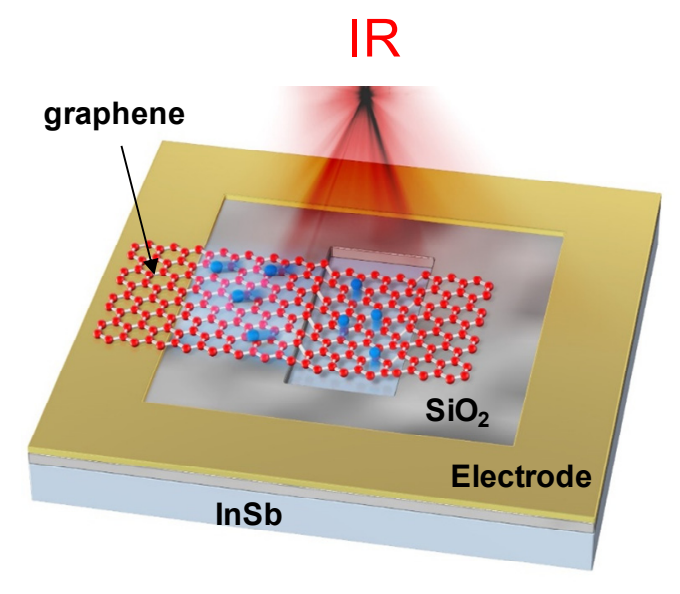

(b)

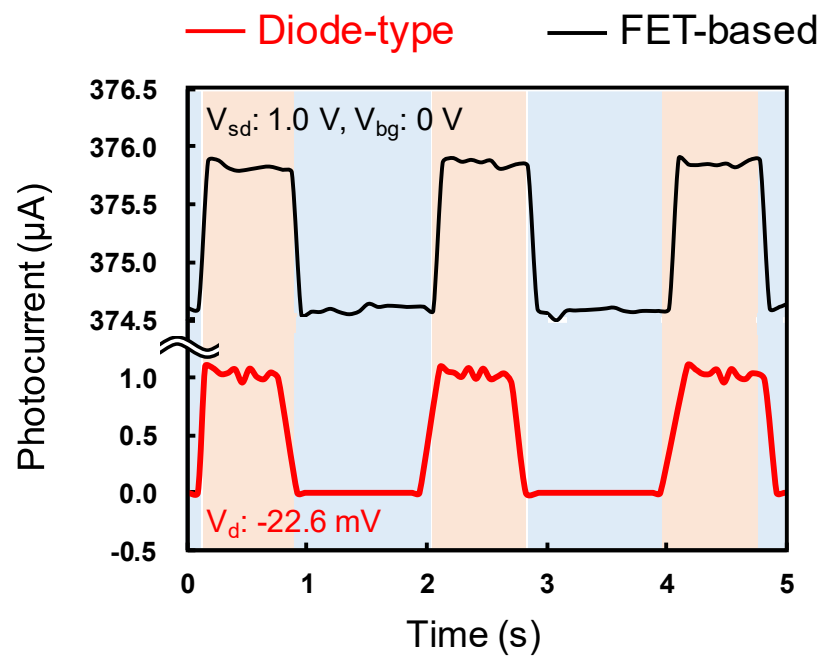

Figure 5. (a) Schematic diagram of graphene/InSb heterostructure-based MWIR PD. (b) Comparison of photoresponse between graphene FET-based and diode-type MWIR PDs with photogating (InSb substrate).

As shown in Fig. 5(b), the diode-type MWIR $\mathrm{PD}^{30}$ has the same high responsivity as that of the FET-based MWIR PD but with a much lower dark current (about $10^{-7}$ times). This indicates that the photogating effect was induced in the diode-type PD. 


\section{CONCLUSION}

We developed high-responsivity graphene FET-based and diode-type IR PDs with photogating. The photogating effect is generated by the voltage change induced by a photosensitizer located in the vicinity of graphene. The photogating effect is attributed to graphene's unique properties (e.g., high carrier mobility and atomic thickness). Ultra-high-responsivity graphene FET-based PDs with photogating were demonstrated using $\mathrm{Si}, \mathrm{InSb}$, and $\mathrm{LiNbO}_{3}$ as a photosensitizer in the VIS and NIR, MWIR, and LWIR regions, respectively. Room-temperature operation was realized using the pyroelectric effect of $\mathrm{LiNbO}_{3}$. Although high responsivity was achieved, graphene FET-based PDs produce a large dark current, which is a serious disadvantage for IR sensors and IR image sensors. To overcome this problem, we developed graphene-based diode-type PDs, which comprise a contact region and a graphene channel. In the contact region, graphene and InSb (as a photosensitizer) are in direct contact. The barrier formed by the Fermi level difference between graphene and InSb drastically suppresses the dark current. The graphene channel forms on the insulator layer on the substrate, where photogating can occur. As a result, both a low dark current and high responsivity can be achieved in the MWIR region.

The high responsivity obtained with photogating is proportional to carrier mobility. Therefore, high-quality graphene is required for high performance. Turbostratic graphene is a promising candidate for obtaining high carrier mobility because it has the same high carrier mobility as that of mono-layer graphene and suppresses the influence of the insulator layer below the graphene channel ${ }^{40-42}$. Advanced functional graphene IR PDs, such as those with wavelength- or polarization-selective functions ${ }^{43-45}$, are promising for next-generation advanced functional IR sensors based on graphene metamaterials ${ }^{22,46}$. These functions enable multicolor IR imaging for gas analysis and fire detection ${ }^{47}$ and polarimetric imaging ${ }^{48}$.

The results obtained in this study can be applied to other wavelength ranges, such as the ultraviolet and terahertz regions, and could contribute to the development of novel PDs, image sensors, and optoelectronic devices such as optical switches and waveguides.

\section{ACKNOWLEDGEMENT}

I would like to thank my research team members Mr. Masaaki Shimatani, Dr. Shoichiro Fukushima and Dr. Satoshi Okuda at Mitsubishi Electric Corporation Advanced Technology R\&D Center, and our collaborators Prof. Kazuhiko Matsumoto, Dr. Yasushi Kanai and Dr. Takao Ono at Osaka University. This work was partially supported by the Innovative Science and Technology Initiative for Security Grant Number JPJ004596, ATLA, Japan.

\section{REFERENCES}

[1] Novoselov, K. S., Geim, A. K., Morozov, S. V., Jiang, D., Zhang, Y., Dubonos, S. V., Grigorieva, I. V. and Firsov, A. A., "Electric field effect in atomically thin carbon films," Science 306(5696), 666-669 (2004).

[2] Castro Neto, A. H., Guinea, F., Peres, N. M. R., Novoselov, K. S. and Geim, A. K., "The electronic properties of graphene," Rev. Mod. Phys. 81(1), 109-162 (2009).

[3] Bonaccorso, F., Sun, Z., Hasan, T. and Ferrari, A. C., "Graphene photonics and optoelectronics," Nat. Photon. 4, 611-622 (2010).

[4] Franklin, A. D., "Nanomaterials in transistors: From high-performance to thin-film applications," Science 349(6249), aab2750 (2015).

[5] Polat, E. O., Balci, O., Kakenov, N., Uzlu, H. B., Kocabas, C. and Dahiya, R., "Synthesis of Large Area Graphene for High Performance in Flexible Optoelectronic Devices," Sci. Rep. 5, 16744 (2015).

[6] Ago, H., Kawahara, K., Ogawa, Y., Tanoue, S., Bissett, M. A., Tsuji, M., Sakaguchi, H., Koch, R. J., Fromm, F., Seyller, T., Komatsu, K. and Tsukagoshi, K., "Epitaxial Growth and Electronic Properties of Large Hexagonal Graphene Domains on $\mathrm{Cu}(111)$ Thin Film," Appl. Phys. Exp. 6(7), 075101 (2013).

[7] Low, T. and Avouris, P., "Graphene Plasmonics for Terahertz to Mid-Infrared Applications," ACS Nano 8(2), 1086-1101 (2014). 
[8] Xia, F., Wang, H., Xiao, D., Dubey, M. and Ramasubramaniam, A., "Two-dimensional material nanophotonics," Nat. Photon. 8(12), 899-907 (2014).

[9] Mueller, T., Xia, F. and Avouris, P., "Graphene photodetectors for high-speed optical communications," Nat. Photon. 4(5), 297-301 (2010).

[10] Cai, X., Sushkov, A. B., Suess, R. J., Jadidi, M. M., Jenkins, G. S., Nyakiti, L. O., Myers-Ward, R. L., Li, S., Yan, J., Gaskill, D. K., Murphy, T. E., Drew, H. D. and Fuhrer, M. S., "Sensitive room-temperature terahertz detection via the photothermoelectric effect in graphene," Nat. Nanotechnol. 9(10), 814-819 (2014).

[11] Engel, M., Steiner, M., Lombardo, A., Ferrari, A. C., Löhneysen, H. v., Avouris, P. and Krupke, R., "Lightmatter interaction in a microcavity-controlled graphene transistor," Nat. Commun. 3, 906 (2012).

[12] Furchi, M., Urich, A., Pospischil, A., Lilley, G., Unterrainer, K., Detz, H., Klang, P., Andrews, A. M., Schrenk, W., Strasser, G. and Mueller, T., "Microcavity-integrated graphene photodetector," Nano Lett. 12(6), 27732777 (2012).

[13] Fang, Z., Liu, Z., Wang, Y., Ajayan, P. M., Nordlander, P. and Halas, N. J., "Graphene-antenna sandwich photodetector," Nano Lett. 12(7), 3808-3813 (2012).

[14] Ogawa, S., Fujisawa, D. and Ueno, M., "Effect of graphene on plasmonic metasurfaces at infrared wavelengths," AIP Adv. 3(11), 112127 (2013).

[15] Ogawa, S., Shimatani, M., Fukushima, S., Okuda, S. and Matsumoto, K., "Graphene on metal-insulator-metalbased plasmonic metamaterials at infrared wavelengths," Opt. Express 26(5), 5665 (2018).

[16] Shimatani, M., Ogawa, S., Fukushima, S., Okuda, S., Inoue, K. and Matsumoto, K., "Multispectral graphene infrared photodetectors using plasmonic metasurfaces," Proc. SPIE 11002, 1100224 (2019).

[17] Yao, Y., Shankar, R., Rauter, P., Song, Y., Kong, J., Loncar, M. and Capasso, F., "High-Responsivity MidInfrared Graphene Detectors with Antenna-Enhanced Photocarrier Generation and Collection," Nano Lett. 14, 3749-3754 (2014).

[18] Ju, L., Geng, B., Horng, J., Girit, C., Martin, M., Hao, Z., Bechtel, H. A., Liang, X., Zettl, A., Shen, Y. R. and Wang, F., "Graphene plasmonics for tunable terahertz metamaterials," Nat. Nanotechnol. 6(10), 630-634 (2011).

[19] Freitag, M., Low, T., Zhu, W., Yan, H., Xia, F. and Avouris, P., "Photocurrent in graphene harnessed by tunable intrinsic plasmons," Nat. Commun. 4, 1951 (2013).

[20] Ogawa, S., Fukushima, S. and Shimatani, M., "Graphene Plasmonics in Sensor Applications: A Review," Sensors 20(12), 3563 (2020).

[21] Guo, Q., Yu, R., Li, C., Yuan, S., Deng, B., García De Abajo, F. J. and Xia, F., "Efficient electrical detection of mid-infrared graphene plasmons at room temperature," Nat. Mater. 17(11), 986-992 (2018).

[22] Ogawa, S., Shimatani, M., Fukushima, S., Okuda, S. and Kimata, M., "Multilayer graphene metamaterial absorbers for high-performance middle- to long-wavelength infrared detection," Proc. SPIE 11407, 114071I (2020).

[23] Yan, J., Kim, M. H., Elle, J. A., Sushkov, A. B., Jenkins, G. S., Milchberg, H. M., Fuhrer, M. S. and Drew, H. D., "Dual-gated bilayer graphene hot-electron bolometer," Nat. Nanotechnol. 7(7), 472-478 (2012).

[24] Liu, N., Tian, H., Schwartz, G., Tok, J. B., Ren, T. L. and Bao, Z., "Large-area, transparent, and flexible infrared photodetector fabricated using P-N junctions formed by N-doping chemical vapor deposition grown graphene," Nano Lett. 14(7), 3702-3708 (2014).

[25] Konstantatos, G., Badioli, M., Gaudreau, L., Osmond, J., Bernechea, M., Garcia De Arquer, F. P., Gatti, F. and Koppens, F. H., "Hybrid graphene-quantum dot phototransistors with ultrahigh gain," Nat. Nanotechnol. 7(6), 363-368 (2012).

[26] Goossens, S., Navickaite, G., Monasterio, C., Gupta, S., Piqueras, J. J., Pérez, R., Burwell, G., Nikitskiy, I., Lasanta, T., Galán, T., Puma, E., Centeno, A., Pesquera, A., Zurutuza, A., Konstantatos, G. and Koppens, F., "Broadband image sensor array based on graphene-CMOS integration," Nat. Photon. 11(6), 366-371 (2017).

[27] Ni, Z., Ma, L., Du, S., Xu, Y., Yuan, M., Fang, H., Wang, Z., Xu, M., Li, D., Yang, J., Hu, W., Pi, X. and Yang, D., "Plasmonic Silicon Quantum Dots Enabled High-Sensitivity Ultrabroadband Photodetection of GrapheneBased Hybrid Phototransistors," ACS Nano 11(10), 9854-9862 (2017).

[28] An, X., Liu, F., Jung, Y. J. and Kar, S., "Tunable graphene-silicon heterojunctions for ultrasensitive photodetection," Nano Lett. 13(3), 909-916 (2013).

[29] Riazimehr, S., Kataria, S., Gonzalez-Medina, J. M., Wagner, S., Shaygan, M., Suckow, S., Ruiz, F. G., Engström, O., Godoy, A. and Lemme, M. C., "High Responsivity and Quantum Efficiency of Graphene/Silicon Photodiodes Achieved by Interdigitating Schottky and Gated Regions," ACS Photonics 6(1), 107-115 (2018). 
[30] Fukushima, S., Shimatani, M., Okuda, S., Ogawa, S., Kanai, Y., Ono, T., Inoue, K. and Matsumoto, K., "Low dark current and high-responsivity graphene mid-infrared photodetectors using amplification of injected photocarriers by photo-gating," Opt. Lett. 44(10), 2598-2601 (2019).

[31] Fukushima, S., Shimatani, M., Okuda, S. and Ogawa, S., "Carrier density modulation and photocarrier transportation of graphene/InSb heterojunction middle-wavelength infrared photodetectors," Opt. Eng. 59(9), $097101(2020)$.

[32] Shimatani, M., Fukushima, S., Okuda, S. and Ogawa, S., "High-performance graphene/InSb heterojunction photodetectors for high-resolution mid-infrared image sensors," Appl. Phys. Lett. 117(17), 173102 (2020).

[33] Shimatani, M., Ogawa, S., Fujisawa, D., Okuda, S., Kanai, Y., Ono, T. and Matsumoto, K., "Giant Dirac point shift of graphene phototransistors by doped silicon substrate current," AIP Adv. 6(3), 035113 (2016).

[34] Fukushima, S., Shimatani, M., Okuda, S., Ogawa, S., Kanai, Y., Ono, T. and Matsumoto, K., "High responsivity middle-wavelength infrared graphene photodetectors using photo-gating," Appl. Phys. Lett. 113(6), 061102 (2018).

[35] Shimatani, M., Ogawa, S., Fukushima, S., Okuda, S., Kanai, Y., Ono, T. and Matsumoto, K., "Enhanced photogating via pyroelectric effect induced by insulator layer for high-responsivity long-wavelength infrared graphene-based photodetectors operating at room temperature," Appl. Phys. Exp. 12(2), 025001 (2019).

[36] Shimatani, M., Fukushima, S., Okuda, S., Ogawa, S., Kanai, Y., Ono, T., Inoue, K. and Matsumoto, K., "Room temperature long-wavelength infrared graphene photodetectors using photogating via the pyroelectric effect," Proc. SPIE 11407, 1140717 (2020).

[37] Fukushima, S., Shimatani, M., Okuda, S., Ogawa, S., Kanai, Y., Ono, T., Inoue, K. and Matsumoto, K., "Photogating for small high-responsivity graphene middle-wavelength infrared photodetectors," Opt. Eng. 59(3), 037101 (2020).

[38] Ogawa, S., Shimatani, M., Fukushima, S., Okuda, S., Kanai, Y., Ono, T. and Matsumoto, K., "Broadband photoresponse of graphene photodetector from visible to long-wavelength infrared wavelengths," Opt. Eng. 58(5), 057106 (2019).

[39] Behura, S. K., Wang, C., Wen, Y. and Berry, V., "Graphene-semiconductor heterojunction sheds light on emerging photovoltaics," Nat. Photon. 13, 312-318 (2019).

[40] Uemura, K., Ikuta, T. and Maehashi, K., "Turbostratic stacked CVD graphene for high-performance devices," Jpn. J. Appl. Phys. 57(3), 030311 (2018).

[41] Shimatani, M., Yamada, N., Fukushima, S., Okuda, S., Ogawa, S., Ikuta, T. and Maehashi, K., "Highresponsivity turbostratic stacked graphene photodetectors using enhanced photogating," Appl. Phys. Exp. 12(12), 122010 (2019).

[42] Okuda, S., Shimatani, M., Fukushima, S., Ogawa, S., Ikuta, T. and Maehashi, K., "First-principles calculation of electronic structure of turbostratic graphene for high-responsivity infrared detection with enhanced photogating effect," Proc. SPIE 11407, 114071J (2020).

[43] Ogawa, S. and Kimata, M., "Wavelength- or Polarization-Selective Thermal Infrared Detectors for Multi-Color or Polarimetric Imaging Using Plasmonics and Metamaterials," Materials 10(5), 493 (2017).

[44] Ogawa, S., Komoda, J., Masuda, K. and Kimata, M., "Wavelength selective wideband uncooled infrared sensor using a two-dimensional plasmonic absorber," Opt. Eng. 52(12), 127104 (2013).

[45] Ogawa, S., Masuda, K., Takagawa, Y. and Kimata, M., "Polarization-selective uncooled infrared sensor with asymmetric two-dimensional plasmonic absorber," Opt. Eng. 53(10), 107110 (2014).

[46] Ogawa, S., Shimatani, M., Fukushima, S., Okuda, S. and Kimata, M., "Graphene-based Salisbury screen metasurfaces at infrared wavelengths," Proc. SPIE 11002, 110022A (2019).

[47] Vollmer, M. and Mollmann, K.-P., [Infrared Thermal Imaging: Fundamentals, Research and Applications], Wiley-VCH, Weinheim, Germany, (2010).

[48] Tyo, J. S., Goldstein, D. L., Chenault, D. B. and Shaw, J. A., "Review of passive imaging polarimetry for remote sensing applications," Appl. Opt. 45(22), 5453-5469 (2006). 